\title{
MAPPINGS BETWEEN EUCLIDEAN SPACES THAT ARE ONE TO ONE OVER THE IMAGE OF A DENSE SUBSET
}

\author{
MLADEN BESTVINA AND JOHN J. WALSH ${ }^{1,2}$
}

\begin{abstract}
Examples are constructed that include, for $n>m \geq 2$, proper surjective maps $f: \mathbf{R}^{n} \rightarrow \mathbf{R}^{m}$ that are one to one over the image of a dense subset $D \subset \mathbf{R}^{n}$; i.e., the restriction of $f$ yields a homeomorphism from $f^{-1} f(D)$ onto $f(D)$. A routine Baire Category argument establishes that, for any $\sigma$ compact subset $F \subset \mathbf{R}^{n}$ which $f$ maps onto $\mathbf{R}^{m}, \operatorname{Int}(F) \neq \emptyset$ and, hence, $\operatorname{dim} F=n$.
\end{abstract}

0. Introduction. In general, a deficient point of a map $f: X \rightarrow Y$ refers to a point $y \in Y$ for which some characteristic of $f^{-1}(y)$ does not reflect global properties of the map $f$. One type of deficiency, studied by Church and Timourian $[\mathbf{C T}]$ and Honkapohja $\left[\mathbf{H o}_{1}, \mathbf{H o}_{2}\right]$, arises for maps between closed oriented $n$ manifolds, say $f: N^{n} \rightarrow M^{n}$, a deficient point being a $y \in M^{n}$ for which the cardinality of $f^{-1}(y)$ is less than $\mid$ degree $f \mid$. No such deficiency arises for $n=1$ and a result of Hopf $[\mathbf{H}]$ constrains the number of deficient points to be finite for $n=2$; but, for $n \geq 3$, the deficient points can be dense. Specifically, it is shown in [Wa] that, for $n \geq 3$ and each integer $d$, there is a map $f: S^{n} \rightarrow S^{n}$ having degree $d$ that is one to one over the image of a dense subset (i.e., there is a dense subset $D \subset S^{n}$ for which the restriction of $f$ is a homeomorphism from $f^{-1}(f(D))$ onto $f(D))$. While the examples presented in this paper and discussed below exhibit similar behavior, the notion of deficiency differs. The setting is proper surjective maps $f: N^{n} \rightarrow M^{m}$ between manifolds with $n>m$ and a deficient point is a $y \in M^{m}$ for which $\operatorname{dim} f^{-1}(y)<n-m$. A fundamental result from dimension theory detects that the set of nondeficient points is dense [HW, p. 92]. The most extreme type of deficiency occurs at points where $f^{-1}(y)$ consists of exactly one point, and we shall concentrate on displaying this densely.

The first author describes in [Be] examples that include maps between spheres $f: S^{n} \rightarrow S^{m}$ for $n>m \geq 4$ that are one to one over the image of a dense subset. These examples differ dramatically from those found herein, for the maps in [Be] are monotone (i.e., each point inverse $f^{-1}(y)$ is connected) and are, in many cases, essential! (For example, the nontrivial element of $\pi_{n+1}\left(S^{n}\right) \cong \mathbf{Z} / 2 \mathrm{Z}$ for $n \geq 4$ has such a representation.) The techniques used are reminiscent of those in [Wa] and contrast sharply with the elementary techniques we employ to establish

Received by the editors July 6, 1983.

1980 Mathematics Subject Classification. Primary 54B15, 54C05; Secondary 54N99.

Key words and phrases. Deficient points, dendrite, manifold, stable values, upper semicontinuous decomposition.

${ }^{1}$ Research partially supported by a National Science Foundation Grant

${ }^{2}$ Alfred P. Sloan Fellow 
MAIN TheOREM. Suppose that $N^{n}$ and $M^{m}$ are compact connected PL manifolds with $n>m \geq 2$. Then there is a map $f$ from $N^{n}$ onto $M^{m}$ that is one to one over the image of a dense subset.

The map $f$ is constructed in two stages. First, $\S 1$ contains a description of a monotone map $h$ from $N^{n}$ onto a dendrite $A$ such that, setting $E=$ \{endpoints of $A\}, h^{-1}(E)$ is dense and the restriction of $h$ is a homeomorphism from $h^{-1}(E)$ onto $E$. (Recall from [Why] that a dendrite is a locally connected, uniquely arcwise connected compact metric space. An endpoint is one whose complement is connected.) Second, $\S 2$ describes a surjective "space filling" map $k: A \rightarrow M^{m}$ that is one to one over the image of a dense subset of $E$. Evidently, $f=k \circ h$ satisfies the conclusion of the Main Theorem.

COROLLARY. For each $n>m \geq 2$, there is a proper map $f: \mathbf{R}^{n} \rightarrow \mathbf{R}^{m}$ such that, for any $\sigma$-compact $F \subset \mathbf{R}^{n}$ with $f(F)=\mathbf{R}^{m}, \operatorname{dim} F=n$.

Proof. The restriction of a map from $S^{n}$ to $S^{m}$ as in the Main Theorem produces a map $f$ from $\mathbf{R}^{n}$ to $\mathbf{R}^{m}$ that is one to one over the image of a dense subset, say $D \subset \mathbf{R}^{n}$. A Baire category analysis produces a compact subset $C \subset F$ such that $\operatorname{Int} f(C) \neq \emptyset$. The set $f^{-1}(f(D) \cap \operatorname{Int} f(C))$ is contained in $D \cap C$ and is a nonempty relatively open subset of $D$ and, as $D$ is dense, each point of this set is an interior point of $C$. The inequality $\operatorname{Int} C \neq \emptyset$ reveals that $\operatorname{dim} F=n$.

A search for an example $f: \mathbf{R}^{3} \rightarrow \mathbf{R}^{2}$ as in the Corollary initiated the analysis in this paper as well as that in $[\mathbf{B e}]$. The question of whether such an example existed was circulated by R. D. Edwards at the Regional A.M.S. Meeting in Norman, Oklahoma, in March of 1983, his preamble being that for maps $f: \mathbf{R}^{2} \rightarrow \mathbf{R}^{1}$ (more generally, $\mathbf{R}^{n} \rightarrow \mathbf{R}^{1}$ ) there is always a closed subset $C \subset \mathbf{R}^{\mathbf{2}}$ homeomorphic to $\mathbf{R}^{1}$ with $f(C)=f\left(\mathbf{R}^{2}\right)$.

$\S 3$ establishes that, in searching for maps $f: N^{n} \rightarrow M^{2}, n>2$, that are one to one over the image of a dense subset, there is essentially only one successful route. Any such map must arise as a composition of maps surprisingly similar to the specific maps constructed in the next two sections.

Spaces are separable and metric. A compactum is a compact space. A continuum is a connected compactum.

1. The decomposition $G$. The purpose of this section is to establish that each compact, connected PL $n$-manifold $M^{n}, n \geq 2$, admits an upper semicontinuous decomposition $G$ so that

(a) $M^{n} / G$ is a uniquely arcwise connected, locally connected continuum (i.e., a dendrite);

(b) the set of endpoints of $M^{n} / G$ is dense (an endpoint is a point $g$ for which $\left(M^{n} / G-g\right.$ is connected); and

(c) the set of endpoints of $M^{n} / G$ consists precisely of those elements $g \in G$ that are single points.

The basic building blocks for constructing $G$ are decompositions of the open $n$ cell $B^{n}$ described next. Let $C \subset I=[0,1]$ denote the "middle thirds" Cantor set, let $B^{n}=\left\{x \in E^{n}:|x|<1\right\}$, and let $K$ be the $(n-2)$-skeleton of a triangulation of $S^{n-1}=\left\{x \in E^{n}:|x|=1\right\}$. The nondegenerate elements of a decomposition $H$ of 
$B^{n}$ arise in one of two ways; namely,

1. for each $t \in C \backslash\{0,1\}$ that is not an endpoint of an interval of $I \backslash C$, the $(n-1)$-sphere $\left\{X \in B^{n}:|x|=t\right\}$ is an element of $H$;

2. for each component $(a, b)$ of $I \backslash C$, the set that is the union $\left\{x \in B^{n}:|x|=a\right.$ or $|x|=b\} \cup\{t \cdot x: x \in K$ and $a \leq t \leq b\}$ is an element of $H$.

Setting $N_{H}=\{g \in H: g \neq$ point $\}$, the reader should observe that components of $B^{n} \backslash \bigcup\left\{g \in N_{H}\right\}$ are open $n$-cells except for the origin that is itself a component. Different choices of triangulations of $S^{n-1}$ for specifying $K$ will permit us to insist below that the components of $B^{n} \backslash \bigcup\left\{g \in N_{H}\right\}$ have small diameters.

The nondegenerate elements $N_{G}$ of $G$ arise as a union $N_{H(0)} \cup N_{H(1)} \cup N_{H(2)} \cup \cdots$ where $N_{H(0)}$ possesses a single element that is the $(n-1)$-skeleton of a triangulation of $M^{n}, N_{H(1)}$ is the union of $N_{H(\sigma)}$ 's, one for each open $n$-cell component $\sigma$ of $M^{n} \backslash \bigcup\left\{g \in N_{H(0)}\right\}$, where $H(\sigma)$ is a decomposition of $\sigma$ as described in the preceding paragraph. Recursively, $N_{H(i+1)}$ is the union of $N_{H(\sigma)}$ 's, one for each open $n$-cell component $\sigma$ of $M^{n} \backslash \bigcup\left\{g \in N_{H(i)}\right\}$, where $H(\sigma)$ is a decomposition of $\sigma$ as described in the preceding paragraph. It is a routine exercise to check that each finite union $N_{H(0)} \cup \cdots \cup N_{H(i)}$ forms the nondegenerate elements of an upper semicontinuous decomposition of $M^{n}$ and an equally routine exercise to check the same for $N_{G}$ provided the $H(\sigma)$ 's used to produce $N_{H(i)}$ are chosen so that the diameters of components of $M^{n} \backslash \bigcup\left\{g \in N_{H(0)} \cup \cdots \cup N_{H(i)}\right\}$ are less than $\varepsilon_{i}$ where $\lim _{i \rightarrow \infty} \varepsilon_{i}=0$.

Observations that lead to verification that $G$ satisfies conditions (a)-(c) are that each element of $N_{G}$ separates $M^{n}, M^{n} \backslash N_{G}$ is dense, and given any two elements $g$ and $g^{\prime}$ of $G$ there is a third element $g^{\prime \prime}$ of $G$ separating $g$ and $g^{\prime}$.

2. Space filling maps. A standard method for producing "space filling curves" is to start with a compact, connected subset $A$, consisting of at least two points, of a compact, connected PL $n$-manifold $M^{n}$ and, then, to specify a sequence of maps $\left\{f_{i}: A \rightarrow M^{n}\right\}$ satisfying:

1. $f_{0}$ is the restriction of a homeomorphism of $M^{n}$ so that $f_{0}(A)$ meets the interior of each $n$-simplex of a triangulation $K$ of $M^{n}$;

2. $f_{1}=h_{1} \circ f_{0}$ where $h_{1}$ is a homeomorphism of $M^{n}$ with $h_{1}$ the identity on the $(n-1)$-skeleton of $K$ and $f_{1}(A)$ meeting the interior of each $n$-simplex of the first barycentric subdivision $\beta K$ of $K$; and, recursively,

3. $f_{i}=h_{i} \circ f_{i-1}$ where $h_{i}$ is a homeomorphism of $M^{n}$ with $h_{i}$ the identity on the $(n-1)$-skeleton of $\beta^{i-1} K$ and $f_{i}(A)$ meeting the interior of each $n$-simplex of the $i$ th barycentric subdivision of $\beta^{i} K$ of $K$.

It is a routine matter to check that $f=\lim f_{i}$ is a continuous surjection of $A$ onto $M^{n}$. Given any countable subset $\left\{a_{0}, a_{1}, \ldots\right\} \subset A$ we can arrange that $f^{-1} f\left(a_{i}\right)=a_{i}$ for each $i=1,2, \ldots$ by requiring, in addition, that

4. $f_{0}\left(a_{0}\right)$ is contained in the interior of an $n$-simplex of $K$ and $U\left(a_{0}, 0\right)$ is an open set containing $a_{0}$ and contained in that same $n$-simplex such that the diameter of $f_{0}^{-1}\left(U\left(a_{0}, 0\right)\right)<1$;

5. each of $f_{1}\left(a_{0}\right)$ and $f_{1}\left(a_{1}\right)$ is contained in the interior of an $n$-simplex of $\beta K, f_{1}^{-1}\left(U\left(a_{0}, 0\right)\right) \subset f_{0}^{-1}\left(U\left(a_{0}, 0\right)\right)$, and $U\left(a_{0}, 1\right)$ and $U\left(a_{1}, 1\right)$ are pairwise disjoint open sets containing $f_{1}\left(a_{0}\right)$ and $f_{1}\left(a_{1}\right)$ respectively, with each contained in the interior of an $n$-simplex of $\beta K$, with $U\left(a_{0}, 1\right) \subset U\left(a_{0}, 0\right)$, and with each of $f_{1}^{-1}\left(U\left(a_{0}, 0\right)\right)$ and $f_{1}^{-1}\left(U\left(a_{1}, 1\right)\right)$ having diameter $<\frac{1}{2}$; and, recursively, 
6. each of $f_{i}\left(a_{k}\right), k=0,1, \ldots, i$, is contained in the interior of an $n$-simplex of $\beta^{i} K, f_{i}^{-1}\left(U\left(a_{k}, j\right)\right) \subset f_{j}^{-1}\left(U\left(a_{k}, j\right)\right)$ for $k=0,1, \ldots, i-1$ and $j=k, \ldots, i-1$, and $U\left(a_{0}, i\right), \ldots, U\left(a_{i}, i\right)$ are pairwise disjoint open sets containing $a_{0}, \ldots, a_{i}$, respectively, with each contained in the interior of an $n$-simplex of $\beta^{i} K$, with $U\left(a_{k}, i\right) \subset$ $U\left(a_{k}, i-1\right)$ for $k=0,1, \ldots, i-1$ and with each $f_{i}^{-1}\left(U\left(a_{k}, i\right)\right)$ having diameter less than $1 / i+1$ for $k=0,1, \ldots, i$.

Since any dendrite $A$ can be embedded in a 2-cell $[\mathbf{K u}]$, this establishes that for any compact, connected PL $n$-manifold $M^{n}, n \geq 2$, there is a map $f$ from $A$ onto $M^{n}$ that is one to one over the image of a countable dense subset of the endpoints of $A$.

3. Mappings to 2-manifolds having only unstable values. Contrasting with the examples constructed by the first author in $[\mathbf{B e}]$ that include a map from $S^{n+1}$ to $S^{n}$ that represents the nontrivial element of $\pi_{n+1}\left(S^{n}\right)$, for $n \geq 4$, and that is one to one over the image of a dense subset of $S^{n+1}$, this section establishes that maps from an $n$-manifold, $n>2$, to a 2 -manifold that are one to one over the image of a dense subset have only unstable values. (Recall from $[\mathbf{H W}]$ that, for $f: X \rightarrow Y$, a point $y \in f(X)$ is an unstable value of $f$ provided for each $\varepsilon>0$, there is a map $f^{\prime}: X \rightarrow Y \backslash\{y\}$ that is $\varepsilon$-close to $f$.) In fact, these maps exhibit behavior noticeably like that of the examples constructed in the previous sections.

THEOREM 3.1. If $f: P \rightarrow M^{2}$ is a map from a compact connected ANR $P$ to a 2-manifold and every point of $M^{2}$ is an unstable value of $f$, then in the monotonelight factorization of $f$, say $f=l \circ m$ where $m: P \rightarrow X$ and $l: X \rightarrow M^{2}, X$ is 1-dimensional and $\operatorname{rank} \breve{H}^{1}(X ; \mathbf{Z}) \leq \operatorname{rank} H^{1}(P ; \mathbf{Z})$.

COROLlARY 3.2. If $f: N^{n} \rightarrow M^{2}$ is a map from a compact, connected and simply connected $n$-manifold, $n>2$, onto a 2-manifold and $f$ is one to one over the image of a dense subset of $N^{n}$, then $f=l \circ m$ where $m: N^{n} \rightarrow X$ and $l: X \rightarrow M^{2}$ and $X$ is uniquely arcwise connected, locally connected compactum (i.e., a dendrite).

ProOF OF THEOREM 3.1. We shall exploit the following consequence of a Phragmén-Brouwer type result appearing along with its proof in the Appendix. Setting $k=\operatorname{rank} H^{1}(P ; \mathbf{Z})$, any closed subset $C$ of $P$ has the property that if $C$ separates a pair of points $x, y \in P$, then $C$ contains a closed subset that separates $x$ and $y$ and has at most $k+1$ components. Evidently, any closed subset of $C$ that is minimal with respect to separating $x$ and $y$ has at most $k+1$ components. We proceed and establish four properties of $X$.

(i) Given open subsets $U_{1}$ and $U_{2}$ of $M^{2}$ with $\bar{U}_{1} \cap \bar{U}_{2}=\emptyset$, points $x_{1}, x_{2} \in X$ with $l\left(x_{1}\right) \in U_{1}$ and $l\left(x_{2}\right) \in U_{2}$, and $\varepsilon>0$, there are continua $D_{1}, \ldots, D_{k+1}$ contained in $X \backslash l^{-1}\left(U_{1} \cup U_{2}\right)$, each having diameter $<\varepsilon$, whose union, $D_{1} \cup \cdots \cup D_{k+1}$, separates $x_{1}$ and $x_{2}$.

Because $l$ is light, there exists $\eta>0$ such that any continuum $D \subset X$, whose image $l(D)$ has diameter $<2(k+1) \cdot \eta$, has diameter $<\varepsilon$.

Since every point of $M^{2}$ is an unstable value of $f$, there is an $\eta$-approximation $f^{\prime}: P \rightarrow K$ where $K$ is the 1 -skeleton of a triangulation of $M^{2}$. The group $H=\operatorname{Im}\left\{f_{\#}^{\prime}: \pi_{1}\left(P, p_{0}\right) \rightarrow \pi_{1}\left(K, f^{\prime}\left(p_{0}\right)\right)\right\}$ is a free group, as $\pi_{1}\left(K, f^{\prime}\left(p_{0}\right)\right)$ is free; and, as $f_{*}^{\prime}: H_{1}(P ; \mathbf{Z}) \rightarrow H /[H, H]$ is surjective, $H$ is free on $\leq \operatorname{rank} H_{1}(P ; \mathbf{Z})=$ $\operatorname{rank} H^{1}(P ; \mathbf{Z})=k$ generators. Name $e: \tilde{K} \rightarrow K$ to be the covering space associated 
with the subgroup $H$ of $\pi_{1}\left(K, f^{\prime}\left(p_{0}\right)\right)$ and specify a lift $\tilde{f}^{\prime}: P \rightarrow \tilde{K}$ of $f^{\prime}$. Since $\tilde{K}$ is a 1-dimensional realization of a simplicial complex and since $\operatorname{rank} H_{1}(\tilde{K}) \leq k$ (as the fundamental group of $\tilde{K}$ is free on $\leq k$ generators), given any two points $p, q \in \tilde{K}$ and disjoint open sets $p \in W_{p}$ and $q \in W_{q}$, there are points $b_{1}, b_{2}, \ldots, b_{k+1} \in$ $\tilde{K} \backslash\left(W_{p} \cup W_{q}\right)$ such that the set $\left\{b_{1}, \ldots, b_{k+1}\right\}$ separates $p$ and $q$. (A helpful observation is that if $b$ lies on an $\operatorname{arc}$ in $\tilde{K}$ connecting $p$ and $q$ and $\tilde{K} \backslash\{b\}$ is connected, then $\left.\operatorname{rank} H_{1}(\tilde{K}-\{b\} ; \mathbf{Z})=\operatorname{rank} H_{1}(\tilde{K} ; \mathbf{Z})-1.\right)$

Choose points $a_{1}, a_{2} \in P$ with $m\left(a_{1}\right)=x_{1}$ and $m\left(a_{2}\right)=x_{2}$. Further assume $\eta$ was chosen so that $f^{\prime}\left(a_{1}\right) \in U_{1}$ and $f^{\prime}\left(a_{2}\right) \in U_{2}$ and so that the $\eta$-neighborhods, say $O_{1}$ and $O_{2}$, of $\bar{U}_{1}$ and $\bar{U}_{2}$, respectively, are disjoint. Specify a collection $B=$ $\left\{b_{1}, \ldots, b_{k+1}\right\} \subset \tilde{K} \backslash e^{-1}\left(O_{1} \cup O_{2}\right)$ that separates $\tilde{f}^{\prime}\left(a_{1}\right)$ and $\tilde{f}^{\prime}\left(a_{2}\right)$ and set $V$ equal to the $\eta$-neighborhood of $e(B)$. The choice of $\eta$ yields that $\bar{V} \cap\left(U_{1} \cup U_{2}\right)=\emptyset$ and

$$
f^{-1}(V) \supset\left(f^{\prime}\right)^{-1}(e(B)) \supset\left(\tilde{f}^{\prime}\right)^{-1}(B) ;
$$

hence, $f^{-1}(\bar{V})$ separates $a_{1}$ and $a_{2}$. The Phragmén-Brouwer property established in the Appendix assures that there are (not necessarily distinct) components $E_{1}, E_{2}$, $\ldots, E_{k+1}$ of $f^{-1}(\bar{V})$ whose union separates $a_{1}$ and $a_{2}$. Set $d_{i}=m\left(E_{i}\right)$ for $i=$ $1, \ldots, k+1$.

Observe that the diameter of each $f\left(E_{i}\right)=l\left(D_{i}\right)$ is less than $2(k+1) \cdot \eta$ as $f\left(E_{i}\right)$ is a connected subset of the closed $\eta$-neighborhood of $e(B)=\left\{e\left(b_{1}\right), \ldots, e\left(b_{k+1}\right)\right\}$ and, hence the very first specification in the choice of $\eta$ assures that $D_{i}$ has diameter less than $\varepsilon$. Since the $E_{i}$ 's are components of the $m$-saturated set $f^{-1}(\bar{V})$ and the map $m$ is monotone, the union $D_{1} \cup \cdots \cup D_{k+1}$ separates $x_{1}$ and $x_{2}$.

(ii) Given continua $C_{1}$ and $C_{2}$ in $X$ with $l\left(C_{1}\right) \cap l\left(C_{2}\right)=\emptyset$ and $\varepsilon>0$, there are pairwise disjoint closed subsets $A_{1}, \ldots, A_{k+1}$ contained in $X \backslash\left(C_{1} \cup C_{2}\right)$, each having diameter less than $\varepsilon$, whose union $A_{1} \cup \cdots \cup A_{k+1}$ separates $C_{1}$ and $C_{2}$. Choose points $x_{1} \in C_{1}$ and $x_{2} \in C_{2}$ and specify connected neighborhoods $U_{1}$ of $l\left(C_{1}\right)$ and $U_{2}$ of $l\left(C_{2}\right)$ with $\bar{U}_{1} \cap \bar{U}_{2}=\emptyset$. Using (i) there are pairwise disjoint closed subsets $D_{1}, \ldots, D_{k+1}$ contained in $X \backslash l^{-1}\left(U_{1} \cup U_{2}\right)$, each having diameter $<\varepsilon / k+1$, whose union $D_{1} \cup \cdots \cup D_{k+1}$ separates $x_{1}$ and $x_{2}$. Since $C_{1}$ and $C_{2}$ are connected, the union also separates $C_{1}$ and $C_{2}$. The $A_{i}$ 's can be chosen to be the components of $D_{1} \cup \cdots \cup D_{k+1}$.

(iii) Given $x \in X, \varepsilon>0$, and a countable family $\left\{\left(C_{i}, C_{i}^{\prime}\right): i=1,2, \ldots\right\}$ of pairs of pariwise disjoint continua in $X$ such that $l\left(C_{i}\right) \cap l\left(C_{i}^{\prime}\right)=\emptyset$, there is a neighborhood $V$ of $x$, having diameter less than $\varepsilon$, such that no continuum in Fr $V$ meets both $C_{i}$ and $C_{i}^{\prime}$ for $i=1,2, \ldots$.

Let $U_{1}$ be a neighborhood of $x$ having diameter less than $\varepsilon$ and $V_{1}$ another neighborhood of $x$ with $\bar{V}_{1} \subset U_{1}$. Set $\varepsilon_{1}$ equal to distance of $\bar{V}_{1}$ from $X-U_{1}$. Using (ii), specify pairwise disjoint closed sets $A_{1}, \ldots, A_{k+1}$ having diameter less $\varepsilon_{1}$ whose union separates $C_{1}$ and $C_{1}^{\prime}$. Then $\bar{V}_{1} \cup\left(\bigcup\left\{A_{1}: A_{i} \cap \bar{V}_{1}=\emptyset\right\}\right)$ are disjoint closed sets. Specify a pair of open sets $V_{2} \subset \bar{V}_{2} \subset U_{2}$ with $\bar{V}_{1} \cup\left(\bigcup\left\{A_{i}: A_{i} \cap \bar{V}_{1} \neq \emptyset\right\}\right) \subset V_{2}$ and $\bar{U}_{2} \cap\left[\left(X \backslash U_{1}\right) \cup\left(\bigcup\left\{A_{i}: A_{i} \cap \bar{V}_{1}=\emptyset\right\}\right)\right]=\emptyset$. Set $\varepsilon_{2}$ equal to the distance of $\bar{V}_{2}$ and $X \backslash U_{2}$. Repeat the above construction for $C_{2}$ and $C_{2}^{\prime}$. Proceeding recursively produces nested sequences of open sets $V_{i} \subset \bar{V}_{i} \subset U_{i}$ with $\bar{V}_{i} \subset V_{i+1}$ and $U_{i} \supset \bar{U}_{i+1}$ such that any continuum meeting both $C_{i}$ and $C_{i}^{\prime}$ has to meet either $V_{i+1}$ or $X \backslash U_{i+1}$. Consequently, $V=\bigcup V_{i}$ is the sought after neighborhood of $x$.

(iv) Finally, we establish that $\operatorname{dim} X \leq 1$ and $\operatorname{rank} H^{1}(X ; \mathbf{Z}) \leq \operatorname{rank} H^{1}(P ; \mathbf{Z})$. 
Specify a countable basis for $X$, say $\mathcal{U}$, consisting of connected sets and let $C$ denote the collection of pairs of continua $\left(\bar{U}, \bar{U}^{\prime}\right)$ where $U, U^{\prime} \in U$ and $l(\bar{U}) \cap l\left(\bar{U}^{\prime}\right)=$ $\emptyset$. Given a point $x \in X$ and $\varepsilon>0$, denote by $V$ a neighborhood of $x$ as in (iii): that is, Fr $V$ contains no continuum meeting both $\bar{U}$ and $\bar{U}^{\prime}$ for any pair $\left(\bar{U}, \bar{U}^{\prime}\right) \in \mathcal{C}$. Since $l$ is light, we can conclude that $\operatorname{Fr} V$ contains no nondegenerate continuum. As $\operatorname{Fr} V$ is a totally disconnected compact set, $\operatorname{dim} \operatorname{Fr} V \leq 0$. (If there were a nondegenerate continuum $L \subset \operatorname{Fr} V$, then $l(L) \neq$ point and, consequently, there would be points $x, y \in L$ with $l(x) \neq l(y)$ and a pair $\left(\bar{U}, \bar{U}^{\prime}\right) \in \mathcal{C}$ with $x \in \bar{U}, y \in \bar{U}^{\prime}$, and $l(\bar{U}) \cap l\left(\bar{U}^{\prime}\right)=\emptyset$.)

Since $m$ is monotone, the Vietoris-Begle Mapping Theorem [Sp, p. 344] assures that $m^{*}: \breve{H}^{1}(X ; \mathbf{Z}) \rightarrow H^{1}(P ; \mathbf{Z})$ is one to one and, hence, $\operatorname{rank} \breve{H}^{1}(X ; \mathbf{Z}) \leq$ rank $H^{1}(P, \mathbf{Z})$.

REMARK. The full hypothesis that $P$ is an ANR is not used; that $P$ be a connected, locally connected, and locally simply connected compactum suffices.

ProOF OF COROLLARY 3.2. First, observe that if $f^{-1} f(x)=x$, then $f(x)$ is an unstable value: the restriction of $f$ to the boundary of a "small" $n$-cell neighborhood of $x$ maps into a "small" annular region about $f(x)$ and, as the higher homotopy groups of such a region are trivial, the restriction extends to a map of the $n$-cell into the annular region. Second, the homogeneity properties of $M^{2}$ assure that the unstable values of $f$ are closed, and, as $f$ is one to one over the image of a dense set, every value is unstable. Third, Theorem 3.1 applies to establish that in the monotone-light factorization of $f$, say $f=l \circ m$ where $m: N^{n} \rightarrow X$ and $l: X \rightarrow M^{2}, X$ has dimension one and $\operatorname{rank} \breve{H}^{1}(X ; \mathbf{Z}) \leq \operatorname{rank} H^{1}\left(N^{2} ; \mathbf{Z}\right)=0$ (since $N^{n}$ is simply connected). Fourth, $X$ cannot contain a simple closed curve for $\operatorname{dim} X \leq 1$ would permit the construction of a retraction of $X$ to a simple closed curve contradicting that $\operatorname{rank} \breve{H}^{1}(X ; \mathbf{Z})=0$. Finally, since $X$ is arcwise connected, being a locally connected and connected compactum, $X$ must be uniquely arcwise connected.

Appendix. The following is a general form of the Phragmén-Brouwer property that, in a locally connected and connected compactum having trivial first Cech cohomology, if a closed set separates two points, then some component separates them. A proof is included for completeness.

THEOREM. If $X$ is a locally connected and connected compactum with

$$
\operatorname{rank} \check{H}^{1}(X ; \mathbf{Z}) \leq k
$$

and $C$ is a closed subset of $X$ separating a pair of points $x_{1}, x_{2} \in X$, then there are components $C_{1}, C_{2}, \ldots, C_{k+1}$ of $C$ (not necessarily distinct) whose union $C_{1} \cup \cdots \cup$ $C_{k+1}$ separates $x_{1}$ from $x_{2}$.

PROOF. The cohomology groups appearing below are Čech groups with the integers as coefficient group. Evidently it suffices to establish that a closed set $C$ that is minimal with respect to separating $x_{1}$ from $x_{2}$ has at most $k+1$ components or, equivalently, rank $\tilde{H}^{0}(C) \leq k$. For such a $C$, if $X \backslash C=U \cup V$ is a separation with $x_{1} \in U$ and $x_{2} \in V$, then each $U \cup C$ and $V \cup C$ is connected. Thus the reduced groups $\tilde{H}^{0}(U \cup C)$ and $\tilde{H}^{0}(V \cup C)$ are trivial and $\tilde{H}^{0}(C)$ injects into both $H^{1}(U \cup C, C)$ and $H^{1}(V \cup C, C)$. A Mayer-Vietoris sequence detects the isomorphism

$$
H^{1}(X, C) \cong H^{1}(U \cup C, C) \oplus H^{1}(V \cup C, C)
$$


and, consequently,

$$
\operatorname{rank} H^{1}(X, C) \geq 2 \cdot \operatorname{rank} \tilde{H}^{0}(C) .
$$

The long exact sequence for the pair $(X, C)$ contains

$$
0 \rightarrow \tilde{H}^{0}(C) \rightarrow H^{1}(X, C) \rightarrow H^{1}(X) \rightarrow \cdots,
$$

detecting that $\operatorname{rank} H^{1}(X) \geq \operatorname{rank} H^{1}(X, C)-\operatorname{rank} \tilde{H}^{0}(C)$. The two inequalities detect that $\operatorname{rank} \tilde{H}^{0}(C) \leq \operatorname{rank} H^{1}(X)$.

\section{REFERENCES}

[Be] M. Bestvina, Essential dimension lowering mappings having dense deficiency set, Trans. Amer. Math. Soc. (to appear).

[CT] P. T. Church and J. G. Timourian, Deficient points of maps on manifolds, Michigan Math. J. 27 (1980), 321-338.

[HW] W. Hurewicz and H. Wallman, Dimension theory, Princeton Univ. Press, Princeton, N.J., 1941.

[Ho $]$ M. Honkapohja, Degree and point inverses of mappings on spheres, Ann. Acad. Sci. Fenn. Ser. AI No. 447 (1969), 1-5.

$\left[\mathrm{Ho}_{2}\right] \_$, Degree and point inverses of mappings on generalized manifolds, Ann. Acad. Sci. Fenn. Ser AI Math. Dissertationes No. 4 (1975), 1-19.

[H] H. Hopf, Zur Topologie der Abbildungen von Mannigfaltigkeiten. II, Math. Ann. 102 (1929/1930), $562-623$.

[Ku] K. Kuratowski, Sur le problème des courbes gauches in topologie, Fund. Math. 15 (1930), 271-283.

[Sp] E. H. Spanier, Algebraic topology, McGraw-Hill, New York, 1966.

[Wa] J. J. Walsh, Mappings with dense deficiency set, Michigan Math. J. 28 (1981), 117-127.

[Why] G. T. Whyburn, Analytic topology, Amer. Math. Soc. Colloq. Publ., vol. 28, Amer. Math. Soc., Providence, R.I., 1942.

Department of Mathematics, University of Tennessee, Knoxville, TenNESSEE 37996-1300 